\title{
Correlation between hyperlipidemia and hypertension, mean arterial pressure, pulse pressure among Africans
}

\begin{abstract}
Objective: Cholesterol is an important substance in intermediary metabolism. The correlation between hyperlipidemia and hypertension, mean arterial pressure, pulse pressure is not well characterised in Africans. This paper aim to address this

Methods: This is a pilot study involving 36 hypertensives and 35 normotensives as controls. The blood pressure of each participants was measured using a mercurial sphygmomanometer at heart level in the sitting position. The fasting plasma cholesterol for the hypertensives and control were taken using a cholesterol meter. The JNC 7TH sustained by JNC 8TH Classification of hypertension was used to define hypertension as blood pressure equal to or greater than $140 / 90 \mathrm{mmHg}$. Hypercholesterolemia (hyperlipidemia) was defined as fasting plasma cholesterol above $200 \mathrm{mg} / \mathrm{dl}$. The mean arterial pressure was computed by adding diastolic blood pressure to one third of pulse pressure. The pulse pressure was computed by subtracting diastolic from systolic blood pressure. All measurements were taken during a free medical screening programme in Surulere Lagos, Nigeria, West Africa.
\end{abstract}

Results: The age range among hypertensives was 30 to 81 years while it was 18 to 88 years among normotensive controls. Hyperlipidemia was present in 33.3\% of the hypertensives while it was present in only $17.1 \%$ of the control. We can therefore infer that in this African cohort sample studied though small in size, hyperlipidemia is twice as common in hypertensives in Africa compared to the general African population. Genderwise, hyperlipidemia was commoner among Hypertensives that are female. Further analysis showed that of the 18 subjects $(25.35 \%)$ in the study with hyperlipidemia, $66.6 \%$ had hypertension while $33.3 \%$ were normotensive.

Discussion: Routine screening of hypertensives for hyperlipidemia is supported by this study as 1 out of 3 hypertensives in Africa have hyperlipidemia. This paper finding also support the routine screening for hypertension among subjects with hyperlipidemia as 2 out of 3 with hyperlipidemia had hypertension. There is no correlation between hyperlipidemia and mean arterial pressure in hypertensive Africans according to this study. Non hypertensive Africans with hyperlipidemia tend to have lower mean arterial pressure than the other non hypertensive general population without hyperlipidemia. Hyperlipidemia occurs commonly at low pulse pressure in hypertensives and at wide pulse pressure in non hypertensive Africans.

Conclusion: There is correlation between hyperlipidemia and hypertension and pulse pressure but none between hyperlipidemia and mean arterial blood pressure in African hypertensives according to this pilot study.

Keywords: hyperlipidemia, hypertension, mean arterial pressure, pulse pressure
Volume 5 Issue 6 - 2017

\author{
Adegbenga Ademolu \\ Lagos State University Teaching Hospital Ikeja Lagos, Nigeria \\ Correspondence: Adegbenga Ademolu, Lagos State University \\ Teaching Hospital Ikeja Lagos, 15 Oba Akinkobi Way lkeja Gra \\ Lagos, Nigeria, Tel +2348033575940 , \\ Email ademoluab@yahoo.com
}

Received: October 26, 2017 | Published: November 21, 2017
Abbreviations: FH, familial hypercholesterolemia; CO, cardiac output; MAP, mean arterial pressure; TRR: total peripheral resistance; PP, pulse pressure

\section{Introduction}

Cholesterol is an important substance in intermediary metabolism. Endocrine condition associated with hyperlipidemia include diabetes mellitus (Type 11 especially) and hypothyroidism. Genetic predisposition, cigarette smoking, obesity, poor diet, and a sedentary lifestyle can all lead to hyperlipidemia. ${ }^{1}$ Hyperlipidemia can be acquired or familial.

The diagnosis of familial hypercholesterolemia by genetic testing is hampered by its cost and effectiveness. ${ }^{1}$ in many parts of the world including Africa. Familial Hypercholesterolemia (FH) is a common cause of premature cardiovascular disease and is often undiagnosed in young people. ${ }^{2}$ The principal metabolic causes of atherosclerosis include hyperlipidemia, hypertension, obesity, insulin resistance and diabetes mellitus.

Concerning hyperlipidemia, Subjects with Familial hypercholesterolemia are at increased risk for cardiac events. Therefore, there is an ardent need for the early diagnosis followed by aggressive therapeutic intervention and lifestyle modification. ${ }^{3}$

Three groups have developed clinical diagnostic tools for Familial hypercholesterolemia: the US MedPed Program, the Simon Broome Register Group in the United Kingdom, and the Dutch Lipid Clinic Network, ${ }^{3}$ though analysis of these criteria is not within the scope of this article however it is needful to say that studies on familial hypercholesterolemia using any of the criteria in Africa is not within 
public domain to the best of our knowledge as at the time of writing this article and it will be worth looking into in the near future.

Hypertension in endocrine disorder is well documented in conditions like hypothyroidism, hyperthyroidism, phaeochromocytoma, conns syndrome, cushion syndrome. Hypertension is a frequent feature of endogenous Cushing's syndrome with a prevalence of approximately $80 \%$ in adults. ${ }^{4,5}$ Hypertension is seen much more commonly in ectopic Cushing's (95\%). ${ }^{1}$ However, it is less common in children and adolescents (about 47\%). ${ }^{6}$

Hypertension is the single biggest contributor to the global burden of disease, ${ }^{7,8}$ and is the most common chronic medical condition presenting to primary care physicians in Singapore. ${ }^{9}$ Hypertension and hyperlipidemia coexist in some individuals and in some endocrine condition like hypothyroidism.

It is reported that among individuals aged 40 to 90 years, each $20 / 10 \mathrm{~mm} \mathrm{Hg}$ rise in BP doubles the risk of fatal coronary events. ${ }^{10,11}$ Treating hypertension only reduces CHD risk 25\%. Treating hyperlipidemia in hypertensive patients reduces residual CHD risk $35 \% .^{12}$ while effective treatment of both hypertension and hyperlipidemia would reduce CHD by. $\geq 50 \%$ and decrease residual risk observed when only hypertension is addressed. ${ }^{12}$

Recently a growing body of evidence has indicated that hypercholesterolaemia promotes impairment in several mechanisms implicated in blood pressure control such as nitric oxide bioavailability, renin-angiotensin activity, the sympathetic nervous system, sodium and fluid homeostasis and ion transport/signal transduction. ${ }^{13}$

The global variations in the prevalence of elevated cholesterol among patient with history of hyperlipidemia are associated with country-level economic development and health system indices. ${ }^{14}$

The mean arterial pressure (MAP), as calculated from the systolic and diastolic blood pressures, varies among individuals. ${ }^{15,16}$ The physiologic basis and pathophysiologic implications of mean arterial pressure are well understood. Mean arterial pressure is the product of cardiac output (CO) and total peripheral resistance (TPR), is the time-averaged blood pressure within the arterial circuit, as well as the average pressure in the arteries throughout the cardiac cycle. It is reliant on the elastic properties of arterial walls and the mean volume of blood in the arterial system. ${ }^{17}$ Mean arterial pressure is a critically important cardiovascular variable as it is the average effective pressure that drives blood through the systemic circulation. ${ }^{17}$

Studies on the usefulness of blood pressure as a prognostic factor in cardiovascular disease have more often involved investigations of the levels of diastolic or systolic blood pressure. However, blood pressure may be divided into two other components: steady (mean pressure) and pulsatile (pulse pressure). ${ }^{18}$

Although a large pulse pressure (PP) measured at the brachial artery with use of the sphygmomanometer is not an accurate representation of the proximal aortic pulse pressure, it does suggest a stiffened aorta Such stiffening, through a variety of mechanisms, ${ }^{19,20}$ tends to raise the SBP and lower the DBP. This affects the pulse pressure.

Correlation between hyperlipidemia, hypertension, mean arterial pressure and pulse pressure is not well characterised in Africans and in the world. The aim of this paper is to answer these questions what proportion of Africans Hypertensive have hyperlipidemia? 1s the frequency of hyperlipidemia in hypertensive in Africa the same in both gender? Is there a correlation between hyperlipidemia and mean arterial pressure? Is there correlation between hyperlipidemia and pulse pressure?

This paper also asks the questions what proportion of patient with hypercholesterolemia are hypertensive in Africa? lt is hoped that the level of awareness of coexisting hypercholesterolemia and hypertension will be raised amongst African so as to give way for better management approach.

\section{Methodology}

This is a pilot study involving 36 hypertensives and 35 normotensives as controls. The blood pressure of each participant was measured using a mercurial sphygmomanometer at heart level in the sitting position. The JNC 7TH sustained by JNC 8TH Classification of hypertension was used to define hypertension as $\mathrm{B} / \mathrm{P} \geq 140 / 90 \mathrm{mmHg}$.

The fasting plasma cholesterol for the hypertensives and control were taken using a cholesterol meter. All participants were preinformed to come fasting for the exercise. Hyperlipidemia was defined as fasting plasma cholesterol above $200 \mathrm{mg} / \mathrm{dl}$. All measurements were taken during a free medical screening programme in Surulere Lagos, Nigeria, West Africa. The hypertensive subjects and normotensives subjects were randomly selected hence this is a randomised controlled study.

The mean arterial pressure was computed by using the formular diastolic blood pressure plus one third of pulse pressure. The pulse pressure was computed by subtracting diastolic from systolic blood pressure. Further analysis was with the aid of SPSS statistics 17.0.

\section{Statistical analysis}

By using descriptive statistic in all subjects both hypertensive and control, the mean of the systolic blood pressure was $133 \mathrm{mmHg}$, the median was same as the mode and was $130 \mathrm{mmHg}$. The lowest systolic blood pressure was $84 \mathrm{mmHg}$ while the highest was $270 \mathrm{mmHg}$. The mean of the diastolic was $85.80 \mathrm{mmHg}$, the median was $80 \mathrm{mmHg}$ while the mode was also $80 \mathrm{mmHg}$. The lowest diastolic was $52 \mathrm{mmHg}$ while the highest was $140 \mathrm{mmHg}$. The mean of the PP was $47.42 \mathrm{mmHg}$, the median was $46 \mathrm{mmHg}$ while the mode was $40 \mathrm{mmHg}$, the lowest value was $14 \mathrm{mmHg}$ while the highest was $130 \mathrm{mmHg}$. The mean of MAP was $76.07 \mathrm{mmHg}$, the median was $72.67 \mathrm{mmHg}$ while the mode was $66.67 \mathrm{mmHg}$, The standard deviation was 20.7. The mean of cholesterol was $177.08 \mathrm{mg} / \mathrm{dl}$, the median was $179 \mathrm{mg} / \mathrm{dl}$ while the mode was $140 \mathrm{mg} / \mathrm{dl}$, the standard deviation was 34.5 .

By using the pearson correlation, there is an inverse relationship between hyperlipidemia and pulse pressure as the pearson correlation was -0.011 with a p-value of 0.927 . The pearson correlation between hyperlipidemia and mean arterial pressure was 0.043 with p-value of -0.722 .Using the pearson correlation further showed that the systolic blood pressure was well correlated to mean arterial pressure (0.955), pulse pressure $(0.863)$ and diastolic blood pressure $(0.794)$ in that order with a p-value of less than 0.0001 . These are all shown in Table 1 . 
Table I Correlations between Hypercholesterolemia, Systolic Blood Pressure, Diastolic Blood Pressure, Mean Arterial Presure (MAP) and Pulse Pressure (PP)

\begin{tabular}{|c|c|c|c|c|c|c|}
\hline & & Cholesterol & Systolic & Diastolic & MAP & $\mathbf{P P}$ \\
\hline \multirow{5}{*}{ Cholesterol } & Pearson Correlation & I & .115 & .224 & .043 & -.011 \\
\hline & Sig. (2-tailed) & & .340 & .060 & .722 & .927 \\
\hline & $\begin{array}{l}\text { Sum of Squares and Cross- } \\
\text { products }\end{array}$ & 83309.493 & 7732.732 & 8244.183 & 2148.115 & -488.535 \\
\hline & Covariance & 1190.136 & 110.468 & II 7.774 & 30.687 & -6.979 \\
\hline & $\mathrm{N}$ & 71 & 7I & 71 & 71 & 71 \\
\hline \multirow{5}{*}{ Systolic } & Pearson Correlation & .115 & I & $.794 * *$ & $.955^{* *}$ & $.863^{* *}$ \\
\hline & Sig. (2-tailed) & .340 & & .000 & .000 & .000 \\
\hline & $\begin{array}{l}\text { Sum of Squares and Cross- } \\
\text { products }\end{array}$ & 7732.732 & 54421.831 & 23634.958 & $38580.24 I$ & 30848.662 \\
\hline & Covariance & 110.468 & 777.455 & 337.642 & 551.146 & 440.695 \\
\hline & $\mathrm{N}$ & 71 & 71 & 71 & 71 & 71 \\
\hline \multirow{5}{*}{ Diastolic } & Pearson Correlation & .224 & $.794 * *$ & I & $.578 * *$ & $.378 * *$ \\
\hline & Sig. (2-tailed) & .060 & .000 & & .000 & .001 \\
\hline & $\begin{array}{l}\text { Sum of Squares and Cross- } \\
\text { products }\end{array}$ & 8244.183 & 23634.958 & 16271.239 & 12764.693 & 7387.915 \\
\hline & Covariance & I I 7.774 & 337.642 & 232.446 & 182.353 & 105.542 \\
\hline & $N$ & 71 & 7I & 71 & 71 & 71 \\
\hline \multirow{5}{*}{ MAP } & Pearson Correlation & .043 & $.955 * *$ & $.578 * *$ & I & $.974 * *$ \\
\hline & Sig. (2-tailed) & .722 & .000 & .000 & & .000 \\
\hline & $\begin{array}{l}\text { Sum of Squares and Cross- } \\
\text { products }\end{array}$ & 2148.115 & $38580.24 I$ & 12764.693 & 30010.437 & 25862.153 \\
\hline & Covariance & 30.687 & 551.146 & 182.353 & 428.721 & 369.459 \\
\hline & $\mathrm{N}$ & 71 & 71 & 71 & 71 & 71 \\
\hline \multirow{5}{*}{ PP } & Pearson Correlation & -.011 & $.863^{* *}$ & $.378 * *$ & $.974 * *$ & I \\
\hline & Sig. (2-tailed) & .927 & .000 & .001 & .000 & \\
\hline & $\begin{array}{l}\text { Sum of Squares and Cross- } \\
\text { products }\end{array}$ & -488.535 & 30848.662 & 7387.915 & 25862.153 & 23499.324 \\
\hline & Covariance & -6.979 & 440.695 & 105.542 & 369.459 & 335.705 \\
\hline & $N$ & 71 & 71 & 71 & 71 & 71 \\
\hline
\end{tabular}

**Correlation is significant at the 0.01 level (2-tailed).

\section{Results}

A total of 36 hypertensives with age range $30-81$ years and 35 normotensives controls with age range 18-88years were involved in the study. Of the hypertensives $25 \%$ (9) were male and $75 \%$ (27) were female. Among the control normotensive 37\% (13) were male while $63 \%$ (22) were females. The mean age of the hypertensives was 52.86 years while the mean age of the control was 41.51 years.
Hypercholesterolemia was present in $33.3 \%$ (12 subjects) of the hypertensives while it was present in only $17.1 \%$ (6) of the control see Figure 1.

Genderwise, hypercholesterolemia was commoner among hypertensives that are female, $40.74 \%$ ( 11 females) of the hypertensive female population compared to $11.1 \%$ among hypertensive male population see Figure 2. 


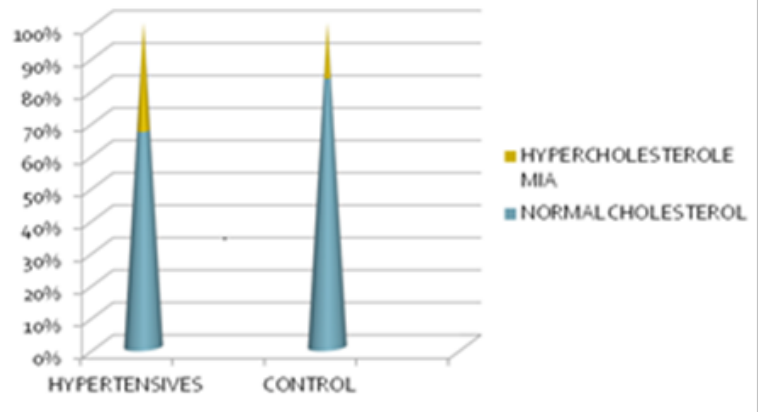

Figure I Percentage distribution of Hypercholesterolemia among African Hypertensive's and Control $(n=7 \mathrm{I})$.

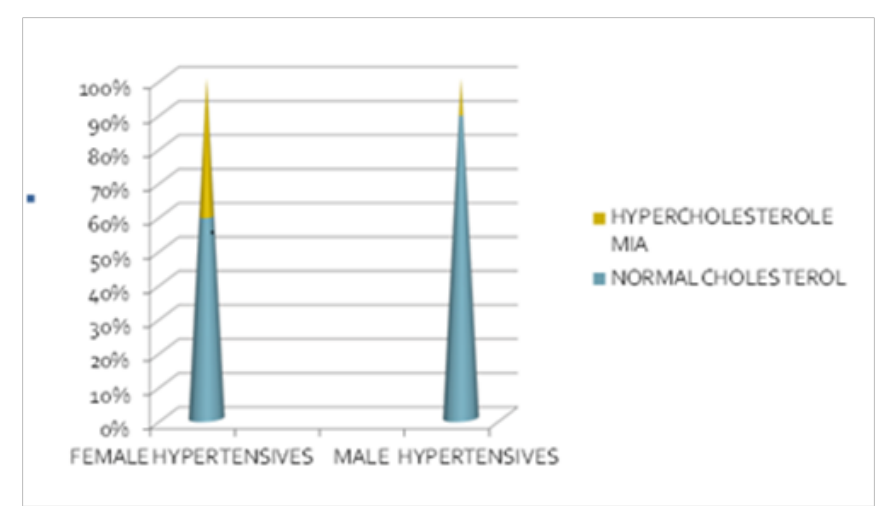

Figure 2 Sex distribution of Hypercholesterolemia among African Hypertensives $(n=36)$.

Further analysis showed that of the 18 subjects $(25.35 \%$ of the population studied) in the study with hypercholesterolemia (both control and hypertensives) $66.6 \%$ (12subjects) have hypertension while $33.3 \%(6)$ were normotensive which translate that 2 out of every 3 with hypercholesterolemia had hypertension.

The relationship between hypercholesterolemia and mean arterial pressure is not linear among African, this is true for both hypertensives and control group in this study. Furthermore among all the hypertensives in this study, the average of mean arterial pressure was $113.98 \mathrm{mmHg}$ while among African hypertensives with hypercholesterolemia the average of mean arterial pressure was $113.75 \mathrm{mmHg}$.

Among all the non hypertensive control, the average of mean arterial pressure was $89.16 \mathrm{mmHg}$ while among the non hypertensives with hypercholesterolemia the average of mean arterial pressure was $87.33 \mathrm{mmHg}$. Hypercholesterolemia occurs at both low and wide pulse pressure in African hypertensives but with those occurring at lower pulse pressure in the majority. Hence there is association between hypercholesterolemia and low pulse pressure in hypertensive Africans. Hypercholesterolemia tend to occur at wider pulse pressure in non hypertensives compared to hypertensive Africans however higher values of hypercholesterolemia does not equate to higher pulse pressure. Normal cholesterol level does not equate to low pulse pressure, as some with normal cholesterol had wide pulse pressure. No linear relationship between cholesterol level (whether normal or not) and pulse pressure. There are other cardiovascular risk factors in the subjects studied for instance diabetes mellitus but the analysis of this is not part of the objective of this study.

\section{Discussion}

The routine screening of hypertensives for hypercholesterolemia is supported by this study as 1 out of 3 hypertensives in Africa have hypercholesterolemia, the dearth of literature on this subject among Africans within the continent limits our understanding of the true picture of hypercholesterolemia among hypertensives in Africa,it may be more than 1 in 3 in some African populations perhaps.

Since hypercholesterolemia was present in $33.3 \%$ of the hypertensives while it was present in only $17.1 \%$ of the control meaning hypercholesterolemia is twice as common among hypertensives compared to the normotensive controls, we can therefore infer that in this African cohort sample studied though small in size, hypercholesteroleamia is twice as common in hypertensives in Africa compared to the general African population.

Accurate determination of MAP is important in the calibration of pressure waveforms for calculating central blood pressure. Currently, a precise, individualized measurement of mean arterial pressure can be obtained only with intra-arterial measurements of blood pressure or with applanation tonometry. ${ }^{15}$

The gold standard for determining MAP would be intra-arterial measurements of systolic blood pressure and diastolic blood pressure however because of its invasive nature such an approach is not feasible in some studies on mean arterial pressure. As a gold standard for non-invasive pressure measurements, the second best solution is applanation tonometry. ${ }^{15}$ However adding $1 / 3$ of the pulse pressure to the diastolic blood pressure is the most frequently used method for calculating mean arterial pressure.

Since among all the hypertensives in this study, the average of mean arterial pressure was $113.98 \mathrm{mmHg}$ while among african hypertensives with hypercholesterolemia the average of mean arterial pressure was $113.75 \mathrm{mmHg}$, this suggest that hypercholesterolemia does not have additional effect on mean arterial pressure among hypertensives in Africa hence there is no correlation between hypercholesterolemia and mean arterial pressure. This has not been documented in any African study based literature on this issue and my study is the first to point this out to the best of my knowledge. A similar study is hereby recommended among African Americans, Hispanics, Native Americans, Latinos, Europeans, Asians and all ethnic groups whether this scientific fact that there is no correlation between hypercholesterolemia and mean arterial pressure is reproducible in other population.

As shown earlier in the result section, among all the non hypertensive control, the average of mean arterial pressure was $89.16 \mathrm{mmHg}$ while among the non hypertensives with hypercholesterolemia the average of mean arterial pressure was $87.33 \mathrm{mmHg}$, hence non hypertensive Africans with hypercholesterolemia tend to have lower mean arterial pressure than the other non hypertensive general population without hypercholesterolemia.

The majority of African hypertensives with hypercholesterolemia in this study had low pulse pressure. However the presence of hypercholesterolemia also at wide pulse pressure points to the fact that there are other co-morbidities in these hypertensives that affects pulse pressure.

These paper findings also support the routine screening for hypertension among subjects with hypercholesterolemia as 2 out of 3 with hypercholesterolemia had hypertension. 
With the newly released definition of hypertension now 130/80 according to the American College of Cardiology and American Heart Association, ${ }^{21}$ it will be interesting to know if this new definition will change the findings in this paper if used compared to the JNC 8th definition of 140/90 used in this study. This is a subject for future research.

\section{Conclusion}

The frequency of hypercholesterolemia among Africans that are hypertensive is more than in the general African population. Similarly majority of Africans with hypercholesterolemia are hypertensive. There is no correlation between hypercholesterolemia and mean arterial pressure in africans. Hypercholesterolemia occurs at low and wide pulse pressures in Africans. Since hypertension and hypercholesterolemia are both risk factors for coronary heart disease, the diagnosis of one should lead to a search for the other as they could coexist in Africans, this will help to reduce the overall mortality rates from coronary heart disease.

There is correlation between hypercholesterolemia and hypertension and pulse pressure but none between hypercholesterolemia and mean arterial blood pressure in African hypertensives. Same study is suggested in other races and ethnic groups.

\section{Acknowledgments}

None.

\section{Conflicts of interest}

The author declares that there are no conflicts of interest.

\section{Funding}

None.

\section{References}

1. Fahed AC, Nemer GM. Familial Hypercholesterolemia: The Lipids or the Genes? Nutr Metab (Lond). 2011;8(1):23.

2. Shoulders CC, EL Jones, RP Naoumova. Genetics of familial combined hyperlipidemia and risk of coronary heart disease. Human Molecular Genetics. 2004;13(Suppl 1):R149-R160.

3. Al-Rasadi K, Al-Waili K, Al-Sabti HA, et al. Criteria for Diagnosis of Familial Hypercholesterolemia: A Comprehensive Analysis of the Different Guidelines, Appraising their Suitability in the Omani Arab Population. Oman Med J. 2014;29(2):85-91.

4. Singh Y, Kotwal N, Menon AS. Endocrine hypertension-Cushing's syndrome. Indian J Endocrinol Metab. 2011;15(Suppl 4):S313-S316.

5. Stewart PM, Walker BR, Holder F. II beta-hydroxysteroid dehydrogenase activity in Cushing's syndrome: Explaining the mineralocorticoid excess state of the ectopic adrenocorticotropin syndrome. J Clin Endocrinol
Metab. 1995;80(12):3617-3620.

6. Magiakou MA, Mastorakos G, Zachman K, et al. Blood pressure in children and adolescents with Cushing's syndrome before and after surgical cure. J Clin Endocrinol Metab. 1997;82(6):1734-1738.

7. Puar TH, Mok Y, Debajyoti R, et al. Secondary hypertension in adults. Singapore Med J. 2016;57(5):228-232.

8. Poulter NR, Prabhakaran D, Caulfield M. Hypertension. Lancet. 2015;386(9995):801-812.

9. Ministry of Health Singapore. Primary Care Survey. 2010.

10. Olafiranye $\mathrm{O}$, Zizi F, Brimah $\mathrm{P}$, et al. Management of Hypertension among Patients with Coronary Heart Disease. Int $J$ Hypertens. 2011;2011:653903.

11. Lewington S, Clarke R, Qizilbash N, et al. Age-specific relevance of usual blood pressure to vascular mortality: a meta-analysis of individual data for one million adults in 61 prospective studies. The Lancet 2012;360(9349):1903-1913

12. Brent ME, Jiexiang Li, Suparna Q, et al. Blood Pressure and Cholesterol Control in Hypertensive Hypercholesterolemic Patients: NHANES 1988-2010. Circulation. 2013;128(1):29-41.

13. Andrei CS, Jose Augusto S Barreto-Filho. Hypercholesterolaemia and its potential role in the presentation and exercerbation of Hypertension. Br J Cardiol. 2004;11(4):292-299.

14. Venkitachalam L, Wang K, Porath A, et al. Global variation in the Prevalence of Elevated Cholesterol in Outpatients with Established Vascular Disease or3 Cardiovascular Risk Factors According to National Indices of Economic Development and Health System Performance. Circulation. 2012;125(15):1858-1869.

15. Vos J, Vincent HH, Verhaar MC, et al. Inaccuracy in Determining Mean Arterial Pressure With Oscillometric Blood Pressure Techniques. Am J Hypertens. 2013;26(5):624-629.

16. Bos WJ, Verrij E, Vincent HH, et al. How to assess mean blood pressure properly at the brachial artery level. J Hypertens. 2007;25(4):51-755.

17. John BH, Matthew CM, Karen CK, et al. An Alternative and Preferable Measurement to Systolic Blood Pressure (SBP) in Patients for Hypotension Detection During Hemapheresis. Journal of Clinical Apheresis. 2002;17:55-64

18. Benetos A, Safar M, Rudnichi A, et al. Pulse Pressure.A Predictor of Long-term Cardiovascular Mortality in a French Male Population. Hypertension. 1997;30(6):1410-1415.

19. Nichols WV, O'Rourke MF. McDonald's Blood Flow in Arteries: Theoretic, Experimental, and Clinical Principles, $3^{\text {rd }}$ edn, London/ Melbourne: E Arnold, UK. 1990;77-142.

20. Safar ME. Pulse pressure in essential hypertension: clinical and therapeutical implications. J Hypertens. 1989;7(10):769-776.

21. New ACC/AHA High Blood Pressure Guidelines Lower Definition of Hypertension. American College of Cardiology. 2017. 